\title{
Direct images of relative pluricanonical bundles
}

\author{
Osamu Fujino
}

\begin{abstract}
We discuss the local freeness and the numerical semipositivity of direct images of relative pluricanonical bundles for surjective morphisms between smooth projective varieties with connected fibers. We give a sought-after semipositivity theorem under the assumption that the geometric generic fiber has a good minimal model.
\end{abstract}

\section{Introduction}

By Griffiths's theory of variations of Hodge structure (see [Gri70]), we have the following result. TheOrem 1.1 (Griffiths). Let $f: X \rightarrow Y$ be a smooth morphism between smooth projective varieties. Then $f_{*} \omega_{X / Y}$ is a nef locally free sheaf.

Before we go further, let us recall the definition of nef (numerically semipositive) locally free sheaves.

Definition 1.2 (Nef locally free sheaves). Let $\mathcal{E}$ be a locally free sheaf of finite rank on a complete algebraic variety $V$. Then $\mathcal{E}$ is called nef if $\mathcal{E}=0$ or $\mathcal{O}_{\mathbb{P}_{V}(\mathcal{E})}(1)$ is nef on $\mathbb{P}_{V}(\mathcal{E})$. A nef locally free sheaf $\mathcal{E}$ was originally called a (numerically) semipositive locally free sheaf in the literature.

More precisely, Griffiths proved that $f_{*} \omega_{X / Y}$ is semipositive in the sense of Griffiths; his result is sharper than Theorem 1.1. Moreover, Berndtsson proved that $f_{*} \omega_{X / Y}$ is semipositive in the sense of Nakano by $L^{2}$ methods (see [Ber09, Theorem 1.2]). Unfortunately, Theorem 1.1 is not so useful for various geometric applications since we need the smoothness of $f$. In [Kaw81], Kawamata proved Theorem 1.3, which is a natural generalization of Theorem 1.1, by using the theory of variations of Hodge structure (see [Kaw81, Theorem 5]).

Theorem 1.3 (Fujita, Zucker, Kawamata, ...). Let $f: X \rightarrow Y$ be a surjective morphism between smooth projective varieties with connected fibers. Then there exists a generically finite morphism $\tau: Y^{\prime} \rightarrow Y$ from a smooth projective variety $Y^{\prime}$ with the following property. Let $X^{\prime}$ be any resolution of the main component of $X \times_{Y} Y^{\prime}$. Then $f_{*}^{\prime} \omega_{X^{\prime} / Y^{\prime}}$ is a nef locally free sheaf, where $f^{\prime}: X^{\prime} \rightarrow X \times_{Y} Y^{\prime} \rightarrow Y^{\prime}$.

For the details of Kawamata's original approach and various generalizations, see [Fujin04, Theorems 3.1, 3.4, and 3.9], [FF14, Theorems 1.1 and 1.3], and [FFS14, Corollary 2 and Theorems 2 and 3]. Theorem 1.3 has already played a crucial role in the study of higher-dimensional

Received 28 January 2015, accepted in final form 26 April 2015.

2010 Mathematics Subject Classification 14D06 (primary), 14E30 (secondary).

Keywords: nef, semipositivity, pluricanonical bundles, good minimal models, weak semistable reduction, effective freeness.

This journal is (c) Foundation Compositio Mathematica 2016. This article is distributed with Open Access under the terms of the Creative Commons Attribution Non-Commercial License, which permits non-commercial reuse, distribution, and reproduction in any medium, provided that the original work is properly cited. For commercial re-use, please contact the Foundation Compositio Mathematica.

The author was partially supported by Grant-in-Aid for Young Scientists (A) 24684002 from JSPS. 


\section{DiRECT IMAGES OF RELATIVE PLURICANONICAL BUNDLES}

algebraic varieties. For some geometric applications, we have to treat $f_{*} \omega_{X / Y}^{\otimes m}$ or $f_{*}^{\prime} \omega_{X^{\prime} / Y^{\prime}}^{\otimes m}$, where $m$ is a positive integer. It is well known that Viehweg proved that $f_{*} \omega_{X / Y}^{\otimes m}$ is always weakly positive for every positive integer $m$ in Theorem 1.3 (see [Vie83, Theorem III]). His original proof of the weak positivity of $f_{*} \omega_{X / Y}^{\otimes m}$ uses his mysterious covering trick and Theorem 1.3 (see [Vie83, $\left.\S 5\right]$ ).

Theorem 1.1 can be generalized as follows.

TheOREm 1.4. Let $f: X \rightarrow Y$ be a smooth morphism between smooth projective varieties. Then $f_{*} \omega_{X / Y}^{\otimes m}$ is a nef locally free sheaf for every positive integer $m$.

We give a proof of Theorem 1.4 based on Siu's invariance of plurigenera (see [Siu02, Corollary 0.2] and [Pău07, Theorem 1]) and the effective freeness in [PS14] (see [PS14, Theorem 1.4]). Note that Siu's invariance of plurigenera is not Hodge theoretic. It is a very clever application of the Ohsawa-Takegoshi $L^{2}$ extension theorem. We have no Hodge-theoretic characterization of $f_{*} \omega_{X / Y}^{\otimes m}$ in Theorem 1.4 when $m \geqslant 2$. By Theorems 1.3 and 1.4, it is natural to consider the following.

Conjecture 1.5 (Semipositivity of direct images of relative pluricanonical bundles). Let $f$ : $X \rightarrow Y$ be a surjective morphism between smooth projective varieties with connected fibers. Then there exists a generically finite morphism $\tau: Y^{\prime} \rightarrow Y$ from a smooth projective variety $Y^{\prime}$ with the following property. Let $X^{\prime}$ be any resolution of the main component of $X \times_{Y} Y^{\prime}$ sitting in the following commutative diagram:

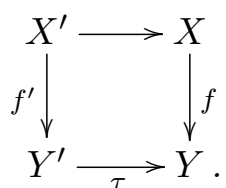

Then $f_{*}^{\prime} \omega_{X^{\prime} / Y^{\prime}}^{\otimes m}$ is a nef locally free sheaf for every positive integer $m$.

Conjecture 1.5 can be seen as a correct formulation of Fujita's very naive conjecture [Fujit78, Conjecture $\mathrm{Wa}_{m}$ ]. Note that [Fujit78] contains 17 conjectures and that "Wa" means 13th in [Fujit78].

The main purpose of this paper is to prove the following result.

Theorem 1.6 (Main theorem). Let $f: X \rightarrow Y$ be a surjective morphism between smooth projective varieties with connected fibers. Assume that the geometric generic fiber $X_{\bar{\eta}}$ of $f: X \rightarrow Y$ has a good minimal model. Then there exists a generically finite morphism $\tau: Y^{\prime} \rightarrow Y$ from a smooth projective variety $Y^{\prime}$ with the following property. Let $X^{\prime}$ be any resolution of the main component of $X \times_{Y} Y^{\prime}$ sitting in the following commutative diagram:

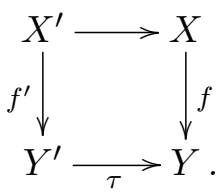

Then $f_{*}^{\prime} \omega_{X^{\prime} / Y^{\prime}}^{\otimes m}$ is a nef locally free sheaf for every positive integer $m$.

We note that $X_{\bar{\eta}}$ has a good minimal model if $\operatorname{dim} X_{\bar{\eta}}-\kappa\left(X_{\bar{\eta}}\right) \leqslant 3$ (see [BCHM10, Lai11], and Theorem 3.8). In particular, $X_{\bar{\eta}}$ has a good minimal model if $X_{\bar{\eta}}$ is of general type. Theorem 1.6 reduces Conjecture 1.5 to the good minimal model conjecture for geometric generic fibers. Of 


\section{O. FuJINO}

course, it is highly desirable to prove Conjecture 1.5 without any extra assumptions. Our proof of Theorem 1.6 is geometric and does not use the theory of variations of Hodge structure. We do not even use $L^{2}$ methods in the proof of Theorem 1.6. Our proof of Theorem 1.6 in this paper is minimal model theoretic. Anyway, Theorems 1.4 and 1.6 strongly support Conjecture 1.5.

Remark 1.7. If $Y$ is a curve in Conjecture 1.5, then Kawamata proved that $f_{*} \omega_{X / Y}^{\otimes m}$ is a nef locally free sheaf for every positive integer $m$ (see [Kaw82, Theorem 1]). We also note that Viehweg's weak positivity of $f_{*} \omega_{X / Y}^{\otimes m}$ (see [Vie83, Theorem III]) implies that $f_{*} \omega_{X / Y}^{\otimes m}$ is nef when $Y$ is a curve.

We sketch the proof of Theorem 1.6 for the reader's convenience.

1.8. Outline of the proof of Theorem 1.6. We take a weak semistable reduction $f^{\dagger}: X^{\dagger} \rightarrow Y^{\prime}$ in the sense of Abramovich-Karu. Then we take a good minimal model $\widetilde{f}: \widetilde{X} \rightarrow Y^{\prime}$ of $f^{\dagger}: X^{\dagger} \rightarrow Y^{\prime}$. Let $P$ be an arbitrary point of $Y^{\prime}$, and let $C$ be a smooth curve on $Y^{\prime}$ such that $P \in C$ and that $C=H_{1} \cap H_{2} \cap \cdots \cap \sim_{\widetilde{X}} H_{\operatorname{dim}} \underset{Y^{\prime}-1}{ }$, where $H_{i}$ is a general very ample Cartier divisor for every $i$. Then we can prove that $\widetilde{X}_{C}=\widetilde{X} \times_{Y^{\prime}} C$ is a normal variety with only canonical singularities. Therefore, we obtain that $\widetilde{f}$ is flat and $\operatorname{dim} H^{0}\left(\widetilde{X}_{y},\left.\mathcal{O}_{\widetilde{X}}\left(m K_{\widetilde{X} / Y^{\prime}}\right)\right|_{\widetilde{X}_{y}}\right)$ is independent of $y \in Y^{\prime}$ for every positive integer $m$. This implies that $f_{*}^{\prime} \omega_{X^{\prime} / Y^{\prime}}^{\otimes m}$ is locally free for every positive integer $m$. Once we establish the local freeness of $f_{*}^{\prime} \omega_{X^{\prime} / Y^{\prime}}^{\otimes m}$, the nefness of $f_{*}^{\prime} \omega_{X^{\prime} / Y^{\prime}}^{\otimes m}$ easily follows from the effective freeness by Popa-Schnell and Viehweg's fiber product trick. As explained above, a key point of the proof of Theorem 1.6 is to construct a good minimal model $\widetilde{f}: \widetilde{X} \rightarrow Y^{\prime}$ which behaves well under the base change by $C \hookrightarrow Y^{\prime}$. Our proof of Theorem 1.6 is not Hodge theoretic.

After the author circulated a preliminary version of this paper, Păun and Takayama informed him of their new preprint [PT14], where they prove various semipositivity theorems by $L^{2}$ methods. Their approach is completely different from ours. For the details, we recommend the reader to see [PT14] (see also Takayama's more recent results in [Tak15]).

We summarize the contents of this paper. In Section 2, we collect some basic definitions and results for the reader's convenience. In Section 3, we discuss the relationship between relative good minimal models and good minimal models of fibers. In Section 4, we prove the local freeness of direct images of relative pluricanonical bundles in Theorem 1.6 after taking a weak semistable reduction. In order to prove the local freeness, we take a relative good minimal model of the weak semistable reduction. Therefore, we need the assumption that the geometric generic fiber has a good minimal model. In Section 5, we prove the numerical semipositivity (nefness) in our main theorem, Theorem 1.6. The proof is an easy application of the effective freeness obtained by Popa-Schnell (see [PS14, Theorem 1.4]) and Viehweg's fiber product trick (see [Vie83, (3.4)]).

We will work over $\mathbb{C}$, the complex number field, throughout this paper. We will freely use the standard notation and results of the minimal model program as in [KM98, Fujin11] and [Fujin14b]. We recommend the reader to see [Mor87, §5] and [Fujin05, Section 5] for the details of Theorem 1.3 and various related topics.

\section{Preliminaries}

In this section, we collect some basic notation and results for the reader's convenience. For the details, see [KM98, Fujin11] and [Fujin14b]. 


\section{DiRECT IMAGES OF RELATIVE PLURICANONICAL BUNDLES}

2.1. Dualizing sheaves and canonical divisors. Let $X$ be a normal quasi-projective variety. Then we put $\omega_{X}=\mathcal{H}^{-\operatorname{dim} X}\left(\omega_{X}^{\bullet}\right)$, where $\omega_{X}^{\bullet}$ is the dualizing complex of $X$, and call $\omega_{X}$ the dualizing sheaf of $X$. We put $\omega_{X} \simeq \mathcal{O}_{X}\left(K_{X}\right)$ and call $K_{X}$ the canonical divisor of $X$. Note that $K_{X}$ is a well-defined Weil divisor on $X$ up to the linear equivalence. Let $f: X \rightarrow Y$ be a morphism between Gorenstein varieties. Then we put $\omega_{X / Y}=\omega_{X} \otimes f^{*} \omega_{Y}^{\otimes-1}$ and call it the relative canonical bundle of $f: X \rightarrow Y$.

2.2. Singularities of pairs. Let $X$ be a normal variety, and let $\Delta$ be an effective $\mathbb{Q}$-divisor on $X$ such that $K_{X}+\Delta$ is $\mathbb{Q}$-Cartier. Let $f: Y \rightarrow X$ be a resolution of singularities. We write

$$
K_{Y}=f^{*}\left(K_{X}+\Delta\right)+\sum_{i} a_{i} E_{i}
$$

and $a\left(E_{i}, X, \Delta\right)=a_{i}$. Note that the discrepancy $a(E, X, \Delta) \in \mathbb{Q}$ can be defined for every prime divisor $E$ over $X$. If $a(E, X, \Delta)>-1$ for every exceptional divisor $E$ over $X$, then $(X, \Delta)$ is called a plt pair. If $a(E, X, \Delta)>-1$ for every divisor $E$ over $X$, then $(X, \Delta)$ is called a $k l t$ pair. In this paper, if $\Delta=0$ and $a(E, X, 0) \geqslant 0$ for every divisor $E$ over $X$, then we say that $X$ has only canonical singularities.

Remark 2.3. Although $\mathbb{R}$-divisors play crucial roles in the recent developments of the minimal model program, we do not use $\mathbb{R}$-divisors in this paper.

We need the following lemma in the proof of the local freeness in the main theorem, Theorem 1.6 .

LEMma 2.4. Let $X$ be a normal variety with only canonical singularities. Then $\mathcal{O}_{X}\left(m K_{X}\right)$ is Cohen-Macaulay for every integer $m$.

Proof. We note that $X$ has only rational singularities when $X$ is canonical. Let $r$ be the smallest positive integer such that $r K_{X}$ is Cartier. Since the problem is local, we may assume that $r K_{X} \sim 0$ by shrinking $X$. If $r=1$, then $\mathcal{O}_{X}\left(m K_{X}\right) \simeq \mathcal{O}_{X}$ for every integer $m$. In this case, $\mathcal{O}_{X}\left(m K_{X}\right)$ is Cohen-Macaulay for every integer $m$ since $X$ has only rational singularities. From now on, we assume $r \geqslant 2$. Let $\pi: \widetilde{X} \rightarrow X$ be the index one cover. Then we have

$$
\pi_{*} \mathcal{O}_{\widetilde{X}}\left(K_{\widetilde{X}}\right) \simeq \bigoplus_{i=1}^{r} \mathcal{O}_{X}\left(i K_{X}\right) .
$$

Since $\widetilde{X}$ has only canonical singularities and $K_{\widetilde{X}}$ is Cartier, $\mathcal{O}_{\widetilde{X}}\left(K_{\widetilde{X}}\right)$ is Cohen-Macaulay. Since $\pi$ is finite, $\mathcal{O}_{X}\left(i K_{X}\right)$ is Cohen-Macaulay for $1 \leqslant i \leqslant r$. By $r K_{X} \sim 0$, we obtain that $\mathcal{O}_{X}\left(m K_{X}\right)$ is Cohen-Macaulay for every integer $m$.

\section{Relative good minimal models}

In this section, we discuss the relationship between relative good minimal models and good minimal models of fibers for the reader's convenience. The results in this section are more or less known to the experts, although they were not stated explicitly in the literature.

Let us recall the definition of sufficiently general fibers.

Definition 3.1 (Sufficiently general fibers). Let $f: X \rightarrow Y$ be a morphism between algebraic varieties. Then a sufficiently general fiber $F$ of $f: X \rightarrow Y$ is a fiber $F=f^{-1}(y)$ where $y$ is any point contained in a countable intersection of Zariski dense open subsets of $Y$. 


\section{O. FuJINO}

A sufficiently general fiber is sometimes called a very general fiber in the literature.

Definition 3.2 (good minimal models). Let $f: X \rightarrow Y$ be a projective morphism between normal quasi-projective varieties. Let $\Delta$ be an effective $\mathbb{Q}$-divisor on $X$ such that $(X, \Delta)$ is klt. A pair $\left(X^{\prime}, \Delta^{\prime}\right)$ sitting in a diagram

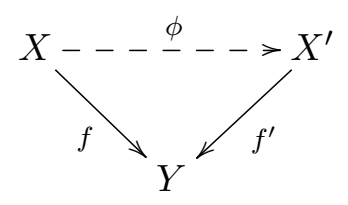

is called a minimal model of $(X, \Delta)$ over $Y$ if

(i) $X^{\prime}$ is $\mathbb{Q}$-factorial;

(ii) $f^{\prime}$ is projective;

(iii) $\phi$ is birational and $\phi^{-1}$ has no exceptional divisors;

(iv) $\phi_{*} \Delta=\Delta^{\prime}$;

(v) $K_{X^{\prime}}+\Delta^{\prime}$ is $f^{\prime}$-nef; and

(vi) $a(E, X, \Delta)<a\left(E, X^{\prime}, \Delta^{\prime}\right)$ for every $\phi$-exceptional divisor $E \subset X$.

Furthermore, if $K_{X^{\prime}}+\Delta^{\prime}$ is $f^{\prime}$-semi-ample, then $\left(X^{\prime}, \Delta^{\prime}\right)$ is called a good minimal model of $(X, \Delta)$ over $Y$. When $Y$ is a point, we usually omit "over $Y^{\prime}$ " in the above definitions. We sometimes simply say that $\left(X^{\prime}, \Delta^{\prime}\right)$ is a relative (good) minimal model of $(X, \Delta)$.

Although Theorem 3.3 holds for klt pairs, we state it for varieties with only canonical singularities for simplicity. Theorem 3.3 is useful and sufficient for our application in this paper.

THEOREM 3.3. Let $f: X \rightarrow Y$ be a projective surjective morphism from a normal quasi-projective variety $X$ with only canonical singularities to a normal quasi-projective variety $Y$ with connected fibers. Then the following conditions are equivalent:

(i) The variety $X$ has a good minimal model over $Y$.

(ii) The variety $X_{\bar{\eta}}$ has a good minimal model, where $X_{\bar{\eta}}$ is the geometric generic fiber of $f: X \rightarrow Y$.

(iii) The variety $F$ has a good minimal model, where $F$ is a sufficiently general fiber of $f: X \rightarrow Y$.

(iv) The variety $G$ has a good minimal model, where $G$ is a general fiber of $f: X \rightarrow Y$.

In order to understand Theorem 3.3, we give some supplementary results.

Theorem 3.4. Let $(X, \Delta)$ be a projective klt pair such that $\Delta$ is a $\mathbb{Q}$-divisor. Then $(X, \Delta)$ has a good minimal model if and only if $K_{X}+\Delta$ is pseudo-effective (equivalently, $\kappa_{\sigma}\left(X, K_{X}+\Delta\right) \geqslant 0$ ) and

$$
\kappa\left(X, K_{X}+\Delta\right)=\kappa_{\sigma}\left(X, K_{X}+\Delta\right),
$$

where $\kappa_{\sigma}$ denotes Nakayama's numerical Kodaira dimension and $\kappa$ denotes Iitaka's $D$-dimension.

Proof. For the proof, see [GL13, Theorem 4.3] or [DHP13, Remark 2.6].

Corollary 3.5. Let $V$ be a smooth projective variety and let $V^{\prime}$ be a normal projective variety with only canonical singularities such that $V$ is birationally equivalent to $V^{\prime}$. Then $V$ has a good minimal model if and only if $V^{\prime}$ has a good minimal model. 
Proof. Note that $\kappa\left(V, K_{V}\right)=\kappa\left(V^{\prime}, K_{V^{\prime}}\right)$ and $\kappa_{\sigma}\left(V, K_{V}\right)=\kappa_{\sigma}\left(V^{\prime}, K_{V^{\prime}}\right)$ hold since $V^{\prime}$ has only canonical singularities. Therefore, we see that $\kappa\left(V, K_{V}\right)=\kappa_{\sigma}\left(V, K_{V}\right)$ if and only if $\kappa\left(V^{\prime}, K_{V^{\prime}}\right)=$ $\kappa_{\sigma}\left(V^{\prime}, K_{V^{\prime}}\right)$. By Theorem 3.4, we have the desired statement.

Lemma 3.6. Let $f: X \rightarrow Y$ be a projective surjective morphism between normal varieties with connected fibers and let $\Delta$ be an effective $\mathbb{Q}$-divisor on $X$ such that $(X, \Delta)$ is klt. Let $X_{\bar{\eta}}$ be the geometric generic fiber of $f: X \rightarrow Y$. We put $\Delta_{\bar{\eta}}=\left.\Delta\right|_{X_{\bar{\eta}}}$. Then we have

$$
\kappa\left(X_{\bar{\eta}}, K_{X_{\bar{\eta}}}+\Delta_{\bar{\eta}}\right)=\kappa\left(F, K_{F}+\left.\Delta\right|_{F}\right)
$$

and

$$
\kappa_{\sigma}\left(X_{\bar{\eta}}, K_{X_{\bar{\eta}}}+\Delta_{\bar{\eta}}\right)=\kappa_{\sigma}\left(F, K_{F}+\left.\Delta\right|_{F}\right),
$$

where $F$ is a sufficiently general fiber of $f: X \rightarrow Y$.

Proof. This is obvious by the definitions of Iitaka's D-dimension $\kappa$ and Nakayama's numerical Kodaira dimension $\kappa_{\sigma}$. For the details, see [Nak04] and [Leh13].

By combining Theorem 3.4 with Lemma 3.6, we have the following result.

Corollary 3.7. Let $f: X \rightarrow Y$ be a projective surjective morphism between normal varieties and let $\Delta$ be an effective $\mathbb{Q}$-divisor on $X$ such that $(X, \Delta)$ is klt. Then $\left(X_{\bar{\eta}}, \Delta_{\bar{\eta}}\right)$ has a good minimal model if and only if $\left(F,\left.\Delta\right|_{F}\right)$ has a good minimal model, where $F$ is a sufficiently general fiber of $f: X \rightarrow Y$.

Proof. This statement is obvious by Theorem 3.4 and Lemma 3.6.

Let us give a proof of Theorem 3.3 for the reader's convenience.

Proof of Theorem 3.3. We divide the proof into several steps.

Step 1: (ii) $\Longleftrightarrow$ (iii). This step is a special case of Corollary 3.7.

Step 2: (iv) $\Longrightarrow$ (iii). This is obvious since a sufficiently general fiber of $f: X \rightarrow Y$ is a general fiber of $f: X \rightarrow Y$.

Step $3:(\mathrm{i}) \Longrightarrow(\mathrm{iv})$. We consider the following commutative diagram:

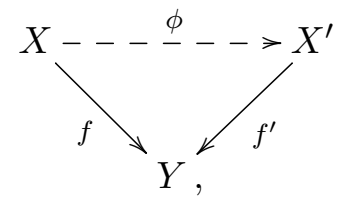

where $f^{\prime}: X^{\prime} \rightarrow Y$ is a good minimal model of $X$ over $Y$. We take a general point $y \in Y$. Let us consider the diagram

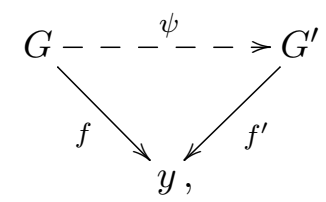

where $G=f^{-1}(y), G^{\prime}=f^{\prime-1}(y)$, and $\psi=\left.\phi\right|_{G}$. Since $y \in Y$ is a general point, the above diagram satisfies the conditions (ii), (iii), (iv), (v), and (vi) in Definition 3.2. Moreover, $K_{G^{\prime}}$ is semi-ample because $K_{X^{\prime}}$ is $f^{\prime}$-semi-ample. If $G^{\prime}$ is not $\mathbb{Q}$-factorial, then we replace $G^{\prime}$ with its small projective $\mathbb{Q}$-factorialization. Then $G^{\prime}$ also satisfies the condition (i) in Definition 3.2 and is a good minimal model of $G$. 


\section{O. FuJINO}

Step 4: (iii) $\Longrightarrow(\mathrm{i})$. This is a special case of [HX13, Theorem 2.12] (see also the proof of [Bir12, Theorem 5.1]).

We have completed the proof of Theorem 3.3.

We close this section with a useful result, which follows from [Lai11, Theorem 4.4] (see also [Bir12, Theorem 1.5] and [HX13, Theorem 2.12]).

Theorem 3.8. Let $X$ be a smooth projective variety with non-negative Kodaira dimension. Then $X$ has a good minimal model if and only if the geometric generic fiber of the Iitaka fibration of $X$ has a good minimal model.

Proof. See [Lai11, Theorem 4.4], [Bir12, Theorem 5.1], and [HX13, Theorem 2.12].

By Theorem 3.8, we know that any smooth projective variety $X$ with $\operatorname{dim} X-\kappa(X) \leqslant 3$ has a good minimal model.

Remark 3.9. In the proof of Theorem 3.8 and Step 4 in the proof of Theorem 3.3, we need the finite generation of canonical rings for (relative) klt pairs, which is established in [BCHM10]. We note that the final step of the proof of the finite generation of canonical rings for klt pairs needs the canonical bundle formula due to Fujino-Mori (see [FM00]). We also note that the canonical bundle formula treated in [FM00] depends on Theorem 1.3. Therefore, our proof of Theorem 1.6 in this paper implicitly uses Theorem 1.3.

\section{Local freeness of $f_{*}^{\prime} \omega_{X^{\prime} / Y^{\prime}}^{\otimes m}$}

In this section, we prove the local freeness of $f_{*}^{\prime} \omega_{X^{\prime} / Y^{\prime}}^{\otimes m}$ in Theorem 1.6 by using minimal model theory and the weak semistable reduction theorem due to Abramovich-Karu (see [AK00]).

Let us start with the proof of the local freeness of $f_{*} \omega_{X / Y}^{\otimes m}$ in Theorem 1.4. It is a direct consequence of Siu's invariance of plurigenera (see [Siu02, Corollary 0.2] and [Pău07, Theorem 1]).

Proof of the local freeness of $f_{*} \omega_{X / Y}^{\otimes m}$ in Theorem 1.4. By [Siu02, Corollary 0.2], we know that

$$
\operatorname{dim} H^{0}\left(X_{y}, \mathcal{O}_{X_{y}}\left(m K_{X_{y}}\right)\right)
$$

is independent of $y \in Y$ for every positive integer $m$ (see also [Pău07, Theorem 1]). By the base change theorem (see [Har77, Chapter III, Corollary 12.9]), this implies that $f_{*} \omega_{X / Y}^{\otimes m}$ is locally free for every $m \geqslant 1$.

Let us recall the following well-known lemma, which is a special case of [Nak86, Corollary 3].

Lemma 4.1 (cf. [Nak86, Corollary 3]). Let $g: V \rightarrow C$ be a projective surjective morphism from a normal quasi-projective variety $V$ to a smooth quasi-projective curve $C$. Assume that $V$ has only canonical singularities and that $K_{V}$ is $g$-semi-ample. Then $R^{i} g_{*} \mathcal{O}_{V}\left(m K_{V}\right)$ is locally free for every $i$ and every positive integer $m$.

Proof. Let $h: V^{\prime} \rightarrow V$ be a resolution of singularities such that $\operatorname{Exc}(h)$ is a simple normal crossing divisor on $V^{\prime}$. We write

$$
K_{V^{\prime}}=h^{*} K_{V}+E,
$$




\section{DiRECT IMAGES OF RELATIVE PLURICANONICAL BUNDLES}

where $E$ is an effective $h$-exceptional $\mathbb{Q}$-divisor. Then we have

$$
\left\lceil m h^{*} K_{V}+E\right\rceil-\left(K_{V^{\prime}}+\left\{-\left(m h^{*} K_{V}+E\right)\right\}\right)=(m-1) h^{*} K_{V} .
$$

We note that the right-hand side is semi-ample over $C$. Therefore,

$$
R^{i}(g \circ h)_{*} \mathcal{O}_{V^{\prime}}\left(\left\lceil m h^{*} K_{V}+E\right\rceil\right)
$$

is locally free for every $i$ and every positive integer $m$ (see, for example, [Fujin11, Theorem 6.3(i)]). On the other hand, we have

$$
R^{i} h_{*} \mathcal{O}_{V^{\prime}}\left(\left\lceil m h^{*} K_{V}+E\right\rceil\right)=0
$$

for every $i>0$ by the relative Kawamata-Viehweg vanishing theorem, and

$$
h_{*} \mathcal{O}_{V^{\prime}}\left(\left\lceil m h^{*} K_{V}+E\right\rceil\right) \simeq \mathcal{O}_{V}\left(m K_{V}\right) .
$$

Therefore, we obtain that

$$
R^{i} g_{*} \mathcal{O}_{V}\left(m K_{V}\right)
$$

is locally free for every $i$ and every positive integer $m$.

Proof of the local freeness of $f_{*}^{\prime} \omega_{X^{\prime} / Y^{\prime}}^{\otimes m}$ in Theorem 1.6. Let us divide the proof into several steps.

Step 1: Weak semistable reduction. By [AK00, Definition 0.1 and Theorem 0.3], there exist a generically finite morphism $\tau: Y^{\prime} \rightarrow Y$ from a smooth projective variety $Y^{\prime}$ and $f^{\dagger}: X^{\dagger} \rightarrow Y^{\prime}$ with the following properties:

(i) The variety $X^{\dagger}$ is a normal projective Gorenstein (see [AK00, Lemma 6.1]) variety which is birationally equivalent to $X \times_{Y} Y^{\prime}$.

(ii) The embeddings $\left(U_{X^{\dagger}} \subset X^{\dagger}\right)$ and $\left(U_{Y^{\prime}} \subset Y^{\prime}\right)$ are toroidal embeddings without selfintersection, with $U_{X^{\dagger}}=\left(f^{\dagger}\right)^{-1}\left(U_{Y^{\prime}}\right)$.

(iii) The morphism $f^{\dagger}:\left(U_{X^{\dagger}} \subset X^{\dagger}\right) \rightarrow\left(U_{Y^{\prime}} \subset Y^{\prime}\right)$ is toroidal and equidimensional.

(iv) All the fibers of the morphism $f^{\dagger}$ are reduced.

In [AK00], the morphism $f^{\dagger}: X^{\dagger} \rightarrow Y^{\prime}$ is said to be weakly semistable and is called a weak semistable reduction of $f: X \rightarrow Y$. For the details of toroidal embeddings and morphisms, see [AK00, Section 1]. We may further assume that $X^{\dagger}$ is $\mathbb{Q}$-factorial (see [AK00, Remark 4.3]). Note that $X^{\dagger}$ has only rational singularities since $X^{\dagger}$ is toroidal. Therefore, $X^{\dagger}$ has only canonical Gorenstein singularities and is Cohen-Macaulay. Thus, we have

$$
f_{*}^{\dagger} \mathcal{O}_{X^{\dagger}}\left(m K_{X^{\dagger} / Y^{\prime}}\right) \simeq f_{*}^{\prime} \omega_{X^{\prime} / Y^{\prime}}^{\otimes m}
$$

for every positive integer $m$. Therefore, it is sufficient to prove that $f_{*}^{\dagger} \mathcal{O}_{X^{\dagger}}\left(m K_{X^{\dagger} / Y^{\prime}}\right)$ is locally free for every positive integer $m$. We also note that $f^{\dagger}$ is flat since $Y^{\prime}$ is smooth, $X^{\dagger}$ is Cohen-Macaulay, and $f^{\dagger}$ is equidimensional (see [Har77, Chapter III, Exercise 10.9] and [AK70, Chapter V, Proposition 3.5]).

Remark 4.2. We may assume that $f^{\dagger}$ is smooth over $U_{Y^{\prime}}$ although we do not need this property in this paper. For the details, see the construction of weak semistable reductions in [AK00].

Step 2: Relative good minimal models. By the assumption of Theorem 1.6 and Corollary 3.5, the geometric generic fiber of $f^{\dagger}: X^{\dagger} \rightarrow Y^{\prime}$ has a good minimal model. Therefore, $f^{\dagger}: X^{\dagger} \rightarrow Y^{\prime}$ has a relative good minimal model $\tilde{f}: \widetilde{X} \rightarrow Y^{\prime}$ by Theorem 3.3. Note that

$$
f_{*}^{\dagger} \mathcal{O}_{X^{\dagger}}\left(m K_{X^{\dagger} / Y^{\prime}}\right) \simeq \widetilde{f}_{*} \mathcal{O}_{\widetilde{X}}\left(m K_{\widetilde{X} / Y^{\prime}}\right)
$$




\section{O. FuJINO}

for every positive integer $m$. Therefore, it is sufficient to prove that $\widetilde{f}_{*} \mathcal{O}_{\widetilde{X}}\left(m K_{\widetilde{X} / Y^{\prime}}\right)$ is locally free for every positive integer $m$.

Step 3: Local freeness via the flat base change theorem. We take an arbitrary point $P \in Y^{\prime}$. We take general very ample Cartier divisors $H_{1}, H_{2}, \ldots, H_{n-1}$, where $n=\operatorname{dim} Y$, such that $C=H_{1} \cap H_{2} \cap \cdots \cap H_{n-1}$ is a smooth projective curve passing through $P$. By [AK00, Lemma 6.2], we see that $X_{C}^{\dagger}=X^{\dagger} \times_{Y^{\prime}} C \rightarrow C$ is weakly semistable. In particular, $X_{C}^{\dagger}$ has only rational Gorenstein singularities (see [AK00, Lemma 6.1]). By adjunction, we see that $\widetilde{X}_{C}=\widetilde{X} \times \times_{Y^{\prime}} C$ is normal and has only canonical singularities. More precisely, $\left(f^{\dagger}\right)^{*} H_{1}=X^{\dagger} \times_{Y^{\prime}} H_{1}=X_{H_{1}}^{\dagger}$ has only rational Gorenstein singularities since $X_{H_{1}}^{\dagger} \rightarrow H_{1}$ is weakly semistable by [AK00, Lemmas 6.1 and 6.2]. In particular, $\left(f^{\dagger}\right)^{*} H_{1}$ has only canonical singularities. Therefore, $\left(X^{\dagger},\left(f^{\dagger}\right)^{*} H_{1}\right)$ is plt by the inversion of adjunction (see [KM98, Theorem 5.50]). So $\left(\widetilde{X}, \widetilde{f}^{*} H_{1}\right)$ is plt by the negativity lemma (see, for example, [KM98, Proposition 3.51]). Thus, $\widetilde{X}_{H_{1}}=\widetilde{X} \times_{Y^{\prime}} H_{1}=\widetilde{f}^{*} H_{1}$ is normal (see [KM98, Proposition 5.51]). By adjunction and the negativity lemma again, $\widetilde{X}_{H_{1}}$ has only canonical singularities. By repeating this process $(n-1)$-times, we obtain that $\widetilde{X}_{C}$ has only canonical singularities. Note that $\widetilde{X}_{C} \rightarrow C$ is equidimensional. Therefore, $\widetilde{f}: \widetilde{X} \rightarrow Y^{\prime}$ is equidimensional by the choice of $C$. Since $\widetilde{X}$ is Cohen-Macaulay and $Y^{\prime}$ is smooth, $\tilde{f}$ is flat (see [Har77, Chapter III, Exercise 10.9] and [AK70, Chapter V, Proposition 3.5]). Moreover, $\mathcal{O}_{\widetilde{X}}\left(m K_{\tilde{X}}\right)$ is flat over $Y^{\prime}$ for every integer $m$ since $\mathcal{O}_{\widetilde{X}}\left(m K_{\tilde{X}}\right)$ is Cohen-Macaulay by Lemma 2.4 and $\tilde{f}$ is equidimensional (see [AK70, Chapter V, Proposition 3.5]). By applying Lemma 4.1 and the base change theorem (see [Har77, Chapter III, Theorem 12.11]) to $\widetilde{X}_{C} \rightarrow C$, we obtain that

$$
\operatorname{dim} H^{0}\left(\widetilde{X}_{y},\left.\mathcal{O}_{\widetilde{X}}\left(m K_{\tilde{X} / Y^{\prime}}\right)\right|_{\widetilde{X}_{y}}\right)
$$

is independent of $y \in Y^{\prime}$ for every positive integer $m$. By the base change theorem (see [Har77, Chapter III, Corollary 12.9]), we obtain that $f_{*}^{\prime} \omega_{X^{\prime} / Y^{\prime}}^{\otimes m} \simeq \widetilde{f}_{*} \mathcal{O}_{\widetilde{X}}\left(m K_{\widetilde{X} / Y^{\prime}}\right)$ is locally free for every positive integer $m$.

We have completed the proof of the local freeness of $f_{*}^{\prime} \omega_{X^{\prime} / Y^{\prime}}^{\otimes m}$.

Remark 4.3. In general, $\widetilde{X}_{y}$ may be non-normal. However, we see that the canonical divisor $K_{\widetilde{X}_{y}}$ is well defined, $\widetilde{X}_{y}$ has only semi log canonical singularities, and $\left.\mathcal{O}_{\widetilde{X}}\left(m K_{\widetilde{X} / Y^{\prime}}\right)\right|_{\widetilde{X}_{y}} \simeq \mathcal{O}_{\widetilde{X}_{y}}\left(m K_{\widetilde{X}_{y}}\right)$ for every positive integer $m$, by adjunction. For the details of semi log canonical singularities and pairs, see [Fujin14a].

In Step 3 in the proof of the local freeness of $f_{*}^{\prime} \omega_{X^{\prime} / Y^{\prime}}^{\otimes m}$ in Theorem 1.6, we have proved the following result.

THEOREM 4.4. Let $\pi: V \rightarrow W$ be a projective surjective morphism between quasi-projective varieties with connected fibers. Assume the following conditions:

(i) The variety $W$ is smooth.

(ii) The embeddings $\left(U_{V} \subset V\right)$ and $\left(U_{W} \subset W\right)$ are toroidal embeddings without self-intersection, with $U_{V}=\pi^{-1}\left(U_{W}\right)$.

(iii) The morphism $f:\left(U_{V} \subset V\right) \rightarrow\left(U_{W} \subset W\right)$ is toroidal and equidimensional.

(iv) All the fibers of the morphism $\pi$ are reduced. 
In this case, $\pi: V \rightarrow W$ is said to be weakly semistable. We know that $V$ has only rational Gorenstein singularities. Let $V^{\prime}$ be a minimal model of $V$ over $W$ sitting in the following diagram.

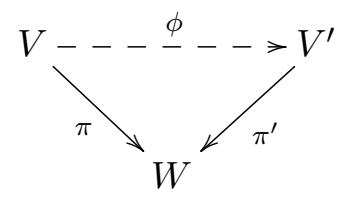

Let $P \in W$ be an arbitrary point, and let $C$ be a smooth curve such that $P \in C$ and that $C=H_{1} \cap H_{2} \cap \cdots \cap H_{\operatorname{dim} W-1}$, where $H_{i}$ is a general smooth Cartier divisor on $W$ for every $i$. Then $V_{C}=V \times_{W} C \rightarrow C$ is weakly semistable and $V_{C}^{\prime}=V^{\prime} \times_{W} C$ is normal and has only canonical singularities. This implies that $\pi^{\prime}: V^{\prime} \rightarrow W$ is equidimensional. In particular, $\pi^{\prime}$ is flat.

Theorem 4.4 seems to be useful for various geometric applications. So we wrote it separately for the reader's convenience. Note that Theorem 4.4 (see also Step 3 in the proof of the local freeness of $f_{*}^{\prime} \omega_{X^{\prime} / Y^{\prime}}^{\otimes m}$ in Theorem 1.6) is a key point of this paper.

\section{Nefness of $f_{*}^{\prime} \omega_{X^{\prime} / Y^{\prime}}^{\otimes m}$}

In this section, we prove that $f_{*}^{\prime} \omega_{X^{\prime} / Y^{\prime}}^{\otimes m}$ in Theorem 1.6 is nef (numerically semipositive) by using [PS14]. We do not use the theory of variations of Hodge structure. Theorem 5.1, which is a key ingredient of this section, follows from [PS14, Theorem 1.4].

THEOREM 5.1. Let $f: X \rightarrow Y$ be a surjective morphism between smooth projective varieties with connected fibers. Let $\mathcal{L}$ be an ample and globally generated line bundle on $Y$ and let $k$ be a positive integer. Then

$$
f_{*} \omega_{X}^{\otimes k} \otimes \mathcal{L}^{\otimes l} \simeq f_{*} \omega_{X / Y}^{\otimes k} \otimes \omega_{Y}^{\otimes k} \otimes \mathcal{L}^{\otimes l}
$$

is generated by global sections for $l \geqslant k(\operatorname{dim} Y+1)$.

Proof. See [PS14, Section 2].

Remark 5.2. Theorem 5.1 holds under the weaker assumption that $X$ is a normal projective variety with only rational Gorenstein singularities. Note that $X$ has only rational Gorenstein singularities if and only if $X$ has only canonical Gorenstein singularities.

LEMMA 5.3. Let $\mathcal{E}$ be a non-zero locally free sheaf of finite rank on a smooth projective variety $V$. Assume that there exists a line bundle $\mathcal{M}$ such that $\mathcal{E}^{\otimes s} \otimes \mathcal{M}$ is generated by global sections for every positive integer $s$. Then $\mathcal{E}$ is nef.

Proof. We put $\pi: W=\mathbb{P}_{V}(\mathcal{E}) \rightarrow V$ and $\mathcal{O}_{W}(1)=\mathcal{O}_{\mathbb{P}_{V}(\mathcal{E})}(1)$. Since $\mathcal{E}^{\otimes s} \otimes \mathcal{M}$ is generated by global sections, $\operatorname{Sym}^{s} \mathcal{E} \otimes \mathcal{M}$ is also generated by global sections for every positive integer $s$. This implies that $\mathcal{O}_{W}(s) \otimes \pi^{*} \mathcal{M}$ is generated by global sections for every positive integer $s$. Thus, we obtain that $\mathcal{O}_{W}(1)$ is nef; equivalently, $\mathcal{E}$ is nef.

Let us prove the nefness of $f_{*} \omega_{X / Y}^{\otimes m}$ in Theorem 1.4.

Proof of the nefness of $f_{*} \omega_{X / Y}^{\otimes m}$ in Theorem 1.4. We take the $s$-fold fiber product

$$
f^{s}: X^{s}=X \times_{Y} X \times_{Y} \cdots \times_{Y} X \rightarrow Y .
$$




\section{O. FuJINO}

Since $f$ is smooth, $X^{s}$ is a smooth projective variety and $f^{s}$ is smooth. We will check

$$
f_{*}^{s} \omega_{X^{s} / Y}^{\otimes m} \simeq \stackrel{s}{\bigotimes} f_{*} \omega_{X / Y}^{\otimes m}
$$

for every positive integer $m$ by induction on $s$. We consider the following commutative diagram:

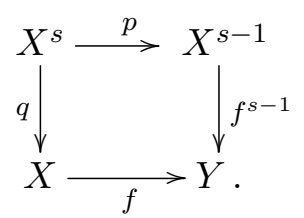

By base change, we have $\omega_{X^{s} / X} \simeq p^{*} \omega_{X^{s-1} / Y}$. Thus we have

$$
\begin{aligned}
\omega_{X^{s} / Y} & \simeq \omega_{X^{s} / X} \otimes q^{*} \omega_{X / Y} \\
& \simeq p^{*} \omega_{X^{s-1} / Y} \otimes q^{*} \omega_{X / Y} .
\end{aligned}
$$

Therefore, by the flat base change theorem (see [Har77, Chapter III, Proposition 9.3]) and the projection formula, we obtain

$$
\begin{aligned}
f_{*}^{s} \omega_{X^{s} / Y}^{\otimes m} & \simeq f_{*}^{s-1} p_{*}\left(p^{*} \omega_{X^{s-1} / Y}^{\otimes m} \otimes q^{*} \omega_{X / Y}^{\otimes m}\right) \\
& \simeq f_{*}^{s-1}\left(\omega_{X^{s-1} / Y}^{\otimes m} \otimes p_{*} q^{*} \omega_{X / Y}^{\otimes m}\right) \\
& \simeq f_{*}^{s-1}\left(\omega_{X^{s-1} / Y}^{\otimes m} \otimes\left(f^{s-1}\right)^{*} f_{*} \omega_{X / Y}^{\otimes m}\right) \\
& \simeq f_{*} \omega_{X / Y}^{\otimes m} \otimes f_{*}^{s-1} \omega_{X^{s-1} / Y}^{\otimes m} \\
& \simeq \bigotimes f_{*}^{s} \omega_{X / Y}^{\otimes m}
\end{aligned}
$$

for every positive integer $m$ and every positive integer $s$ by induction on $s$. Note that $f_{*} \omega_{X / Y}^{\otimes m}$ is locally free for every positive integer $m$ (see Section 4 ). We put $\mathcal{M}=\omega_{Y}^{\otimes m} \otimes \mathcal{L}^{\otimes m(\operatorname{dim} Y+1)}$, where $\mathcal{L}$ is an ample and globally generated line bundle on $Y$. By Theorem 5.1, we obtain that

$$
f_{*}^{s} \omega_{X^{s} / Y}^{\otimes m} \otimes \mathcal{M}
$$

is generated by global sections for every positive integer $s$. This means that

$$
\bigotimes^{s} f_{*} \omega_{X / Y}^{\otimes m} \otimes \mathcal{M}
$$

is generated by global sections for every positive integer $s$. By Lemma 5.3 , we obtain that $f_{*} \omega_{X / Y}^{\otimes m}$ is nef.

In Section 4 , we have already proved that $f_{*}^{\prime} \omega_{X^{\prime} / Y^{\prime}}^{\otimes m}$ is locally free in Theorem 1.6. We conclude by proving the nefness of $f_{*}^{\prime} \omega_{X^{\prime} / Y^{\prime}}^{\otimes m}$ in Theorem 1.6.

Proof of the nefness of $f_{*}^{\prime} \omega_{X^{\prime} / Y^{\prime}}^{\otimes m}$ in Theorem 1.6. By the proof of the local freeness of $f_{*}^{\prime} \omega_{X^{\prime} / Y^{\prime}}^{\otimes m}$ in Section 4, we may assume that $f^{\prime}: X^{\prime} \rightarrow Y^{\prime}$ is weakly semistable. For simplicity, we denote $f^{\prime}: X^{\prime} \rightarrow Y^{\prime}$ by $f: X \rightarrow Y$ in this proof. We take the $s$-fold fiber product

$$
f^{s}: X^{s}=X \times_{Y} X \times_{Y} \cdots \times_{Y} X \rightarrow Y .
$$

Then we see that $X^{s}$ is normal and Gorenstein (cf. [Vie83, Lemma 3.5]). Moreover, $X^{s}$ has only rational singularities because $X^{s}$ is local analytically isomorphic to a toric variety. Therefore, $X^{s}$ 


\section{DiRECT IMAGES OF RELATIVE PLURICANONICAL BUNDLES}

has only canonical singularities. By the same argument as in the proof of the nefness of $f_{*} \omega_{X / Y}^{\otimes m}$ in Theorem 1.4, we obtain

$$
f_{*}^{s} \omega_{X^{s} / Y}^{\otimes m} \simeq \stackrel{s}{\bigotimes} f_{*} \omega_{X / Y}^{\otimes m}
$$

for every positive integer $s$ and every positive integer $m$. By Theorem 5.1 (see also Remark 5.2) and Lemma 5.3, the locally free sheaf $f_{*} \omega_{X / Y}^{\otimes m}$ is nef for every positive integer $m$. This is the same as the proof of the nefness of $f_{*} \omega_{X / Y}^{\otimes m}$ in Theorem 1.4. Anyway, we obtain the desired nefness.

\section{ACKNOWLEDGEMENTS}

The author thanks Yoshinori Gongyo and Professors Shigefumi Mori and Shigeharu Takayama for useful comments. He also thanks Professors Mihai Păun and Shigeharu Takayama for sending him their new preprint [PT14]. Finally, he thanks Jinsong Xu for pointing out a mistake.

\section{REFERENCES}

AK70 A. Altman and S. Kleiman, Introduction to Grothendieck duality theory, Lecture Notes in Math., vol. 146 (Springer-Verlag, Berlin - New York, 1970); http://dx.doi.org/10.1007/ BFb0060932.

AK00 D. Abramovich and K. Karu, Weak semistable reduction in characteristic 0, Invent. Math. 139 (2000), no. 2, 241-273; http://dx.doi.org/10.1007/s002229900024.

BCHM10 C. Birkar, P. Cascini, C. D. Hacon, and J. McKernan, Existence of minimal models for varieties of log general type, J. Amer. Math. Soc. 23 (2010), no. 2, 405-468; http://dx.doi.org/10. 1090/S0894-0347-09-00649-3.

Ber09 B. Berndtsson, Curvature of vector bundles associated to holomorphic fibrations, Ann. of Math. (2) 169 (2009), no. 2, 531-560; http://dx.doi.org/10.4007/annals.2009.169.531.

Bir12 C. Birkar, Existence of log canonical flips and a special LMMP, Publ. Math. Inst. Hautes Études Sci. 115 (2012), no. 1, 325-368; http://dx.doi.org/10.1007/s10240-012-0039-5.

DHP13 J.-P. Demailly, C. D. Hacon, and M. Păun, Extension theorems, non-vanishing and the existence of good minimal models, Acta Math. 210 (2013), no. 2, 203-259; http://dx.doi.org/10. 1007/s11511-013-0094-x.

FF14 O. Fujino and T. Fujisawa, Variations of mixed Hodge structure and semipositivity theorems, Publ. Res. Inst. Math. Sci. 50 (2014), no. 4, 589-661; http://dx.doi.org/10.4171/PRIMS/ 145.

FFS14 O. Fujino, T. Fujisawa, and M. Saito, Some remarks on the semipositivity theorems, Publ. Res. Inst. Math. Sci. 50 (2014), no. 1, 85-112; http://dx.doi.org/10.4171/PRIMS/125.

FM00 O. Fujino and S. Mori, A canonical bundle formula, J. Differential Geom. 56 (2000), no. 1, 167-188; http://projecteuclid.org/euclid.jdg/1090347529.

Fujin04 O. Fujino, Higher direct images of log canonical divisors, J. Differential Geom. 66 (2004), no. 3, 453-479; http://projecteuclid.org/euclid.jdg/1098137840.

Fujin05_L Remarks on algebraic fiber spaces, J. Math. Kyoto Univ. 45 (2005), no. 4, 683-699; http://projecteuclid.org/euclid.kjm/1250281652.

Fujin11_, Fundamental theorems for the log minimal model program, Publ. Res. Inst. Math. Sci. 47 (2011), no. 3, 727-789; http://dx.doi.org/10.2977/PRIMS/50.

Fujin14a , Fundamental theorems for semi log canonical pairs, Algebr. Geom. 1 (2014), no. 2, 194-228; http://dx.doi.org/10.14231/AG-2014-011.

Fujin14b_, Foundation of the minimal model program, 2014, available at https://www.math. kyoto-u.ac.jp/ fujino/foundation1.pdf. 


\section{O. FuJINO}

Fujit78 T. Fujita, Theory of the Kodaira dimension (its past, present, future), Sûgaku 30 (1978), no. 3, 243-254.

GL13 Y. Gongyo and B. Lehmann, Reduction maps and minimal model theory, Compos. Math. 149 (2013), no. 2, 295-308; http://dx.doi.org/10.1112/S0010437X12000553.

Gri70 P.A. Griffiths, Periods of integrals on algebraic manifolds. III. Some global differentialgeometric properties of the period mapping, Publ. Math. Inst. Hautes Études Sci. (1970), no. 38, 125-180; http://www. numdam.org/item?id=PMIHES_1970__38_125_0.

Har77 R. Hartshorne, Algebraic geometry, Grad. Texts in Math., vol. 52 (Springer-Verlag, New York Heidelberg, 1977); http://dx.doi.org/10.1007/978-1-4757-3849-0.

HX13 C. D. Hacon and C. Xu, Existence of log canonical closures, Invent. Math. 192 (2013), no. 1, 161-195; http://dx.doi.org/10.1007/s00222-012-0409-0.

Kaw81 Y. Kawamata, Characterization of abelian varieties, Compos. Math. 43 (1981), no. 2, 253-276; http: //www . numdam.org/item?id=CM_1981_-43_2_253_0.

Kaw82 Kodaira dimension of algebraic fiber spaces over curves, Invent. Math. 66 (1982), no. 1, 57-71; http://dx.doi.org/10.1007/BF01404756.

KM98 J. Kollár and S. Mori, Birational geometry of algebraic varieties, Cambridge Tracts in Math., vol. 134 (Cambridge Univ. Press, Cambridge, 1998); http://dx.doi.org/10.1017/ CB09780511662560.

Lai11 C.-J. Lai, Varieties fibered by good minimal models, Math. Ann. 350 (2011), no. 3, 533-547; http://dx.doi.org/10.1007/s00208-010-0574-7.

Leh13 B. Lehmann, Comparing numerical dimensions, Algebra Number Theory 7 (2013), no. 5, 10651100; http://dx.doi.org/10.2140/ant.2013.7.1065.

Mor87 S. Mori, Classification of higher-dimensional varieties, Algebraic Geometry, Bowdoin, 1985 (Brunswick, Maine, 1985), Proc. Sympos. Pure Math., vol. 46 (Amer. Math. Soc., Providence, RI, 1987), 269-331.

Nak86 N. Nakayama, Invariance of the plurigenera of algebraic varieties under minimal model conjectures, Topology 25 (1986), no. 2, 237-251; http://dx.doi.org/10.1016/0040-9383(86) 90042-X.

Nak04 Z Zariski-decomposition and abundance, MSJ Mem., vol. 14 (Mathematical Society of Japan, Tokyo, 2004).

Pău07 M. Păun, Siu's invariance of plurigenera: a one-tower proof, J. Differential Geom. 76 (2007), no. 3, 485-493; http://projecteuclid.org/euclid.jdg/1180135695.

PS14 M. Popa and C. Schnell, On direct images of pluricanonical bundles, Algebra Number Theory 8 (2014), no. 9, 2273-2295; http://dx.doi.org/10.2140/ant.2014.8.2273.

PT14 M. Păun and S. Takayama, Positivity of twisted relative pluricanonical bundles and their direct images, arXiv:1409.5504.

Siu02 Y.-T. Siu, Extension of twisted pluricanonical sections with plurisubharmonic weight and invariance of semipositively twisted plurigenera for manifolds not necessarily of general type, Complex Geometry (Göttingen, 2000) (Springer, Berlin, 2002), 223-277.

Tak15 S. Takayama, Singularities of Narasimhan-Simha type metrics on direct images of relative pluricanonical bundles, Ann. Inst. Fourier (Grenoble), electronically published on 10 Nov. 2015, http://aif.cedram.org, to appear in print.

Vie83 E. Viehweg, Weak positivity and the additivity of the Kodaira dimension for certain fibre spaces, Algebraic Varieties and Analytic Varieties (Tokyo, 1981), Adv. Stud. Pure Math., vol. 1 (NorthHolland, Amsterdam, 1983), 329-353.

Osamu Fujino fujino@math.kyoto-u.ac.jp

Department of Mathematics, Graduate School of Science, Kyoto University, Kyoto 606-8502, Japan 